\title{
Thioguanine-dependent Light Sensitivity of Perithecial Initiation in Sordaria fimicola
}

\author{
By VIMOL SURAPIPITH AND A. LINDENMAYER \\ Department of Biology, Queens College, City University of New York, \\ Flushing, New York I I367, U.S.A.
}

(Accepted for publication II April 1969)

\begin{abstract}
S UM MARY
Perithecial initiation is strongly inhibited by visible light in less than 18-hr-old hyphae of Sordaria fimicola which are grown in the presence of I $\mu \mathrm{M}$-6-thioguanine. It is known that 6-thioguanine is destroyed when exposed to light in vitro with oxygen and a sensitizing dye like methylene blue is present. The most effective wavelength region for perithecial inhibition is the blue region, indicating that possibly flavins are the photosensitizing compounds in this case. The synthesis of carotenoid and melanin pigments is also affected by blue light in this organism. Another, transient, inhibitory effect on perithecial production is found when light-grown cultures are transferred to darkness. As a consequence of the two light effects, mycelia exposed to alternating light and dark periods in growth tubes develop welldefined zones of perithecia. A simple state-input model is presented in the Appendix to demonstrate how such zonation patterns can be generated on the basis of the light effects and a few other assumptions.
\end{abstract}

\section{INTRODUCTION}

Sordaria fimicola is a homothallic ascomycete in which the development of its sole reproductive structure, the perithecium, is not sensitive to light (Hawker, 1957). Alternating light and darkness affect the spore discharge from mature perithecia of this organism (Ingold \& Dring, 1957; Walkey \& Harvey, 1967) but not their initiation, although the perithecia may attain a larger size under alternating conditions than under continuous light or continuous darkness. In the course of previous work (Lindenmayer \& Schoen, 1967) it was found that certain purine and pyrimidine analogues, and particularly 5 -fluorouracil, could specifically inhibit perithecial initiation in this organism at concentrations at which only slight inhibition of vegetative growth was observed. We also noticed a marked sensitivity of perithecial production to light-dark conditions when the organism was grown in the presence of I to Io $\mu \mathrm{M}-6$ thioguanine or 8-azaguanine. None of the other nucleic acid base analogues produced a similar photodynamic effect. It is known (cf. Shapiro, 1968) that guanine and many of its derivatives are decomposed by visible light in vitro in the presence of molecular oxygen and of catalytic amounts of certain dyes, like methylene blue. It has also been reported (Friedman, 1968) that 6-thioguanine is one of the compounds so affected, but apparently 8-azaguanine is not. The main absorption band of 6-thioguanine at $323 \mathrm{~m} \mu$ (it has no bands in the visible region) disappears as a result of a few minutes of illumination of rather low intensities in the presence of $\mathrm{O}_{2}$ and methylene blue, and no 
other bands appear, indicating the destruction of the purine ring (Friedman, 1968). Since our experiments showed an in vivo photodynamic effect of these compounds, involving presumably a native cellular pigment as the photosensitizing dye, and since the effect consists of the specific suppression of an important morphogenetic process, we decided to investigate this system. But only 6-thioguanine was used in our experiments because 8-azaguanine gave rise to irregular vegetative growth, while 6-thioguanine did not inhibit vegetative growth at all, in fact it stimulated growth somewhat (Lindenmayer \& Schoen, 1967).

\section{METHODS}

A wild-type strain of Sordaria fimicola (Rob.) Ces. et de Not. was obtained from the American Type Culture Collection (strain 145I7).

The culture techniques and the medium, aside from the addition of I $\mu \mathrm{M}-6$-thioguanine before autoclaving, were the same as in the previous work (Lindenmayer \& Schoen, 1967). The medium was a sucrose nitrate medium, containing biotin and thiamine ( $\mathrm{pH} 5.5$ to $6 \cdot 0$ ), and is known to stimulate the production of perithecia (Bretzloff, 1954). To obtain linear surface growth of the fungus, Pyrex screw-cap test tubes ( $15 \mathrm{~cm}$. long, $\mathrm{I} \cdot 2 \mathrm{~cm}$. diam.) were half-filled with the molten agar medium, which was then allowed to solidify while the tubes were in a horizontal position. The average linear growth rate of the fungus was $1 \cdot 5 \mathrm{~cm}$. per day, thus there was enough room for Io days of growth in each tube. All of the experiments were made in a plant growth chamber (Sherer-Gillet model CEL 34-7HT) at constant temperature and illumination. The temperature was kept at $24^{\circ}$. White light was supplied by four Sylvania F48 Tr2-I IO V/CW VHO fluorescent tubes and four $50 \mathrm{~W}$ incandescent bulbs. The culture tubes were placed horizontally at a level $30 \mathrm{~cm}$. below the light sources, and were separated from them by the heavy glass plate enclosing the chamber. The white light intensity at the level of the cultures was estimated as 600 to 800 footcandles. Illumination by light of particular wavelength regions was achieved by using 'Cinemoid' colour filters of known transmission characteristics (supplied by Kliegl Bros., Long Island City, N.Y.). No attempt was made to compensate for the reduction of light intensity under the filters, since even the transmitted intensity was high enough for the effects tested. Cultures were given dark treatment by placing them in light-tight containers in the same growth chamber.

The figures are shown in half natural size with respect to the lengths of the tubes, but their diameters are somewhat further reduced. The dots represent individual perithecia as they were observed at the end of experiment, i.e. at the time when the last boundary line was drawn. Each day at the same hour the advancing front of the colony was marked on the tube, and these boundaries are shown in most figures. Growth took place from left to right. The $L$ and $D$ symbols designate light or darkness to which the advancing mycelium was first exposed in a given interval. It is important to realise that the whole length of each tube was always exposed to light or darkness, thus any given section may have been treated by several cycles of light and dark, and the $L$ or $D$ symbol refers only to the first treatment that section received while the mycelial front was moving through it. Each treatment was administered to pairs or triplets of growth tubes, and each experiment was repeated at least once. The figures show representative samples. 


\section{RESULTS}

First we report on experiments with thioguanine-free cultures, in order to establish the normal behaviour of this organism under alternating light-dark treatments. The results are shown in Fig. I. It is seen (tubes A and B) that perithecia were formed under continuous white light, as well as under continuous darkness (6 days each), although somewhat fewer under light. When cultures were grown in light for 4 days and then transferred to darkness (tube $\mathrm{C}$ ), there was an inhibition of perithecial initiation from

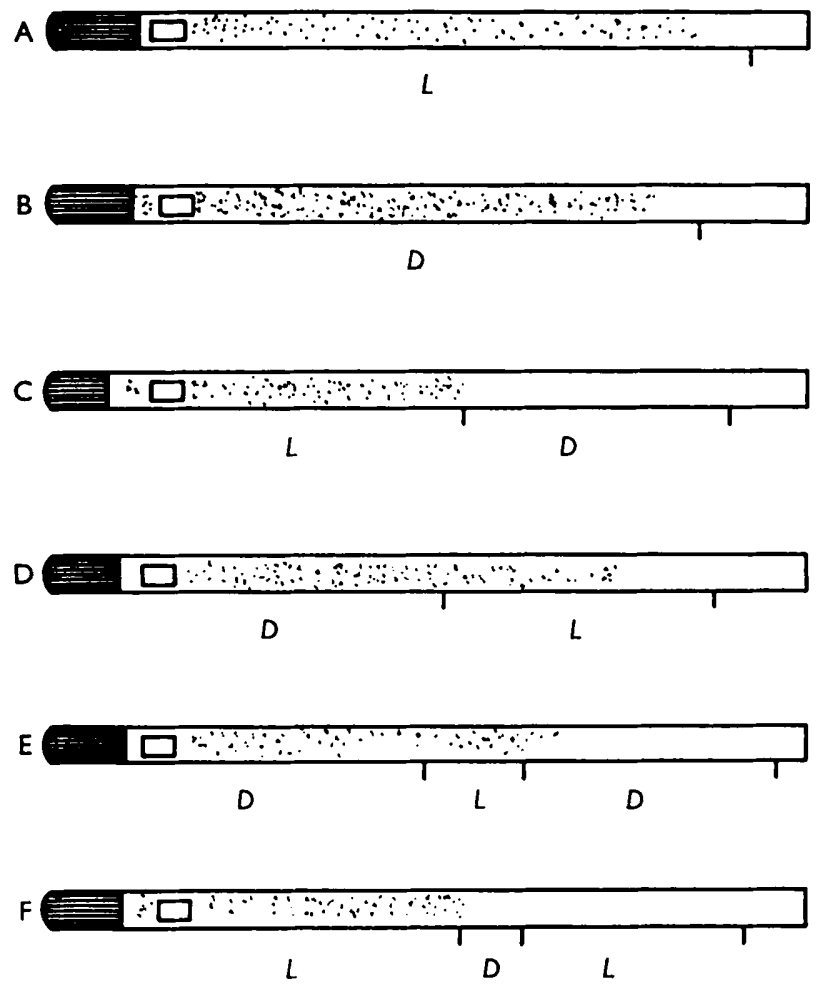

Fig. I. Effects of white light or darkness on thioguanine-free cultures of Sordaria fimicola with respect to production of perithecia. The light or dark treatments were administered to the entire lengths of the growth tubos, beginning or ending when the advancing front of the mycelium was at the vertical lines shown. Inoculum at left. Tubes $E$ and $F$ received $24 \mathrm{hr}$ of light or darknoss, respectively, after 4 days of growth.

the boundary line on. This inhibition was only temporary, however, as evident from other experiments, and extended usually for not longer than I day's growth (in the figure microscopic initials are not shown). When 4 days old dark-grown cultures were transferred to light (tube D), there was no inhibition at the boundary line, in contrast to the findings with thioguanine-grown mycelia. Administering a I-day light period to dark-grown cultures (tube E) gave rise to an inhibition to perithecial formation in the second dark-grown section. One day of darkness given to light-grown cultures (tube F) also showed an inhibition beginning with the start of the dark period. The transient inhibition of perithecial initiation upon passage from light to dark on regular growth medium (and also on thioguanine-containing medium, as is shown below) is a pheno- 
menon not previously reported, which was, however, not investigated any further in this study.

The initial experiment with 6-thioguanine is shown in Fig. 2. In this experiment alternating $24 \mathrm{hr}$ periods of light and darkness were administered to the growth tubes, which in this case were $30 \mathrm{~cm}$. long. The perithecial zonation which developed as a result is shown as it appeared at the end of the experiment (the time of the last boundary position). Each zone was formed in the later part of a light-grown interval, with a rather sharp cut-off at the boundary with the following dark-grown portion. The lightgrown sections were noticeably pink coloured, while the dark-grown ones were colourless at first, with fluffy aerial hyphae present on them.

After the completion of growth, the whole agar content of the tube was allowed to

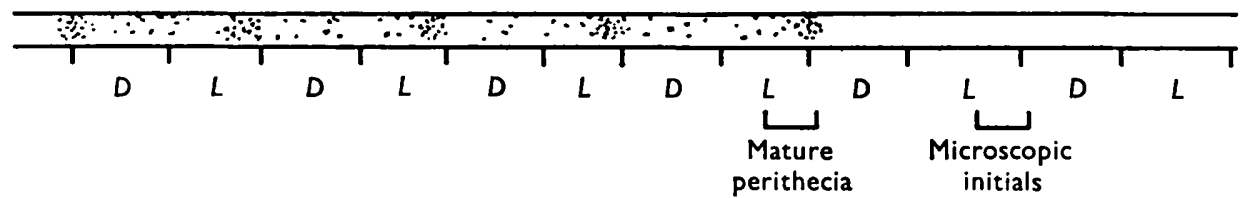

Fig. 2. Perithecial zonation in a culture containing $\mathrm{I} \mu \mathrm{M}-6-$ thioguanine under alternating periods of $24 \mathrm{hr}$ light and $24 \mathrm{hr}$ darkness. The regions where mature perithecia and perithecial initials are indicated were determined by microscopic observations.

slide out (after flaming the entire length of the tube for a short time), and the mycelium of the last 4 days of growth was examined microscopically for perithecial initials. The very young perithecial initials are hook- or coil-shaped structures, as described by Greis (1936). As indicated in Fig. 2, the youngest initials could be seen in the second half of the last but one light-grown section.

From this experiment alone it could be concluded that in thioguanine-grown cultures light is necessary, or darkness is inhibitory, for perithecial initiation. Thioguanine-free cultures grown under an alternating $24 \mathrm{hr}$ light-dark regime do not produce zones. Further experiments demonstrated that far from being stimulatory light was strongly inhibitory for perithecial production in hyphae less than $\mathrm{I} 8 \mathrm{hr}$ old. The reason for the zones arising in the light-grown sections is the $18 \mathrm{hr}$ lag between the initiation of a hypha and that of a perithecium borne by it.

That this is so is demonstrated by the next set of experimental tubes, which were given the same light-dark treatments as those in Fig. I, but contained I $\mu \mathrm{M}-6$-thioguanine. Under continuous light (Fig. 3, tube A), perithecia were almost completely absent, in contrast to the case of the controls (Fig. I, tube A). In continuous darkness (tube B) the number of perithecia formed was, on the other hand, similar to that of the control. When light-grown cultures were switched to the dark (tube C), perithecia appeared on the originally light-grown portion, first near the boundary, then extending more sparsely further back. Following the light-dark boundary there was a short inhibited zone, with normal perithecial density subsequent to that. When dark-grown cultures were switched to light (tube D), perithecial production was inhibited backwards from the boundary line for a distance of about $\mathrm{I} 8 \mathrm{hr}$ growth, and it remained inhibited for the length of the light-grown portion. Administering $24 \mathrm{hr}$ light exposure to dark-grown cultures (tube $\mathrm{E}$ ) resulted in a similar inhibition zone extending back from the boundary line, and also forward from it through half of the light-grown section. Some inhibition was also apparent after the second boundary line, in the 
beginning of the second dark period. Finally, in the reciprocal experiment (tube F), a perithecial zone appears before the first boundary line, on the last section of the light-grown portion. This zone corresponds very closely in its appearance to those in Fig. 2.

It is clear that in thioguanine-grown mycelia perithecial initiation was inhibited by light in hyphae exposed for the first I $8 \mathrm{hr}$ period of their life. On the other hand, if hyphae which were produced in the light were switched to the dark before they were

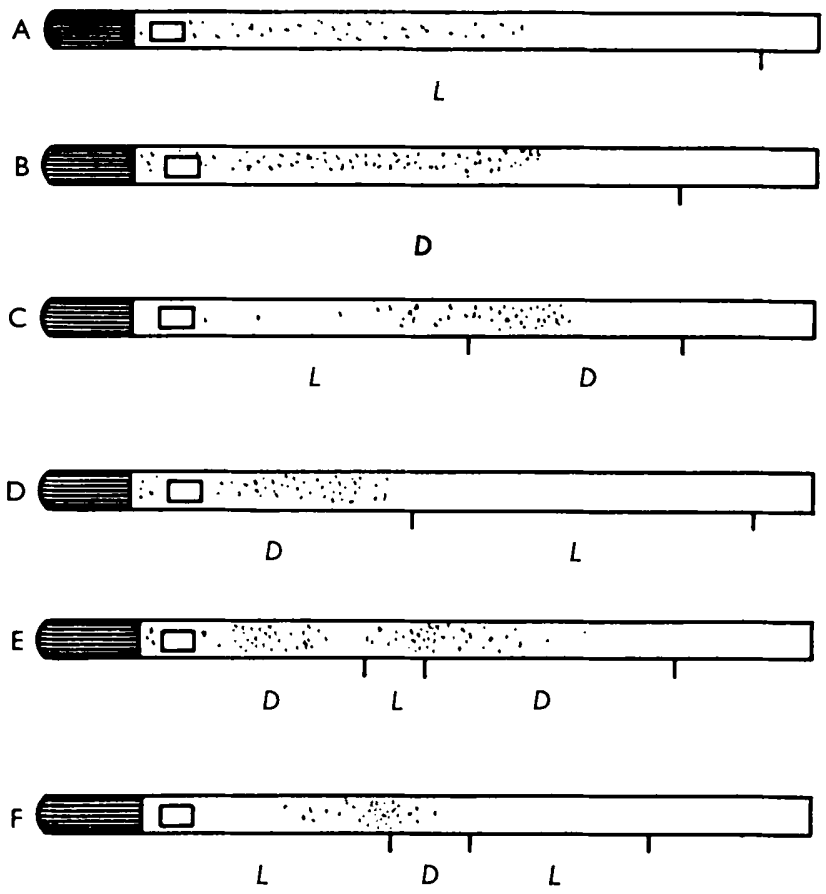

Fig. 3. Effects of white light or darkness on perithecial initiation in cultures containing $1 \mu \mathrm{M}-6$-thioguanine. Same light and dark regimes as in Fig. $\mathrm{I}$.

$18 \mathrm{hr}$ old, they still produced perithecia. The microscopic observations in Fig. 2 confirm this: the last light-grown section cannot have perithecia because it was too young, the preceding dark-grown section cannot have any because most of it was exposed to light when it was still less than $18 \mathrm{hr}$ old, so the first place where perithecia could arise was in the last-but-one light-grown section which received darkness while it was photosensitive. To show that this reasoning can explain the zonation found under alternating $24 \mathrm{hr}$ light-dark regime see computation in Appendix. Another conclusion is that the transient inhibition following the switch from light to dark is observable in thioguanine-containing cultures, although the extent of this inhibition seems to be smaller than in the thioguanine-less cultures.

In order to establish the minimum length of light exposure necessary for the inhibition of perithecia in the presence of thioguanine, various periods of light of constant intensity were administered to dark-grown cultures. These experiments indicated that $8 \mathrm{hr}$ of exposure produced a zone of inhibition as extensive as the one produced by 
$24 \mathrm{hr}$ exposure. Shorter light periods, down to I hr, gave rise to shorter but still welldefined zones of inhibition immediately preceding the boundary. Exposures for 10 and $30 \mathrm{~min}$. did not produce any inhibition.

Experiments were performed to find out which wavelength region was most effective in inhibiting perithecia. Thioguanine-containing cultures were exposed to light, either continuously for several days or for a $24 \mathrm{hr}$ period between longer dark periods, under a series of 'Cinemoid' colour filters. The following filters were used: blueNo. 32 (transmission up to $560 \mathrm{~m} \mu$ and some around $700 \mathrm{~m} \mu$ ), green-No. 39 (transmission between 460 and $580 \mathrm{~m} \mu$ ), yellow-No. I (transmission above $460 \mathrm{~m} \mu$ ), orange-No. 5 A (transmission above $525 \mathrm{~m} \mu$ ) and a combination of red and yellow filters-No. 6 and $\mathrm{I}$ (combined transmission above $580 \mathrm{~m} \mu$, the red filter alone also transmits in the blue region). Blue light was found to be most effective, and, although the total transmission of the blue filter is only $7 \%$ of the incident light, the perithecial inhibition was the same under this filter as under direct white light. The yellow and orange filters gave a certain amount of inhibition but much less than the blue filter. The green and red filters showed the least effect. Thus the blue region of visible light seemed definitely the most effective, and the red region the least. The fact that more inhibition was observed under yellow or orange filters than under green may perhaps be explained by the much lower total transmission of the green filter (12\%) than of the yellow $(90 \%)$ or the orange filter $(39 \%)$. But it is also possible that the photosensitive pigment has two absorption maxima, one in the blue and the other in the yellow region of the spectrum. It should be pointed out here that ultraviolet light could not have been responsible for producing the inhibitory effect, since the cultures were separated from the fluorescent source by a heavy glass plate. It is also clear that the absorbing pigment could not be 6-thioguanine itself, since it does not absorb in the visible region.

Blue light is also responsible for the synthesis of carotenoids in this organism. Their presence was first reported in Sordaria by Ingold \& Hadland (1959), and we could confirm this by obtaining typical carotenoid absorption spectra in intact mycelia with a Cary I4 spectrophotometer (Surapipith, 1969). The mycelia grown under white light or under blue or green filters had very large absorption bands at $430,460,490$ and $520 \mathrm{~m} \mu$, while those grown in the dark, or under yellow, orange or red +yellow filters had only slight peaks in that area, and exhibited absorption bands in addition at $420 \mathrm{~m} \mu$ (the main Soret band of the cytochromes which was masked in the spectra of carotenoid containing cells) and at 320 and $360 \mathrm{~m} \mu$ in the ultraviolet (presumably due to colourless carotene precursors like phytofluene; cf. Goodwin, 1965). The necessity for blue light for carotenoid production in this organism closely resembles the situation in Neurospora crassa, as it was demonstrated by Zalokar (1955).

Another pigment, a green-black melanin-type pigment, is also affected by light in Sordaria fimicola. This pigment is only formed in the dark in the vegetative mycelium (it is presumably the same pigment which makes the perithecia and ascospores black, where it is produced in the light as well). Even $10 \mathrm{~min}$. exposure to white light of thioguanine-grown cultures produced a pigment-less zone which extended back to the mycelium which developed in the two previous days. Thus the light requirement for inhibiting the synthesis of this pigment was much lower than that for inhibiting perithecial production. Blue light was most effective in this case also. Whether there is any connexion between the shutting off of carotenoid production and the start of melanin production in the absence of blue light, is an interesting problem. The black pigment 
of the vegetative mycelium as well as of the perithecia turns yellow when placed in sodium dithionite solution, a strong reducing agent, and turns black again when reoxidized by $\mathrm{H}_{2} \mathrm{O}_{2}$, indicating the presence of quinone groups and conforming to the reactions of allomelanins as described by Nicolaus (1968).

\section{DISCUSSION}

The following assumptions seem to be sufficient to account for the zone patterns obtained with the various light-dark treatments of cultures grown on medium containing 6-thioguanine.

(I) In darkness the newly formed hyphae undergo progressive transformations until they are $18 \mathrm{hr}$ old, when they become determined for bearing perithecia. The first stages of initials are, in fact, observable microscopically on hyphae of about this age, and not on any younger ones.

(2) Under blue light of sufficient intensity the newly formed hyphae undergo progressive changes as well, but these lead after $18 \mathrm{hr}$ to a state in which perithecia cannot be induced even by subsequent dark periods.

(3) If a culture is switched from light to darkness, the hyphal segments which are at that time in intermediate light-grown states change to the age-wise corresponding intermediate dark-grown states, except for short segments at the hyphal tips which become unable to form perithecia. Thus, perithecia will eventually appear as far back as $18 \mathrm{hr}$ growth from the boundary line, but there will be a narrow zone of inhibition immediately after the line.

(4) If a culture is moved from darkness to light, the hyphae in intermediate lightgrown states are transformed into intermediate dark-grown states, but in reverse age order. In other words, the youngest dark-grown state is transformed into the most advanced light-grown state, and the most advanced dark-grown state goes to the least advanced light-grown state. The reverse order needs to be assumed in order to account for the fact that shorter than $18 \mathrm{hr}$ light periods induce proportionately shorter zones of inhibition, that these zones develop immediately next to the boundary line, and that the maturation of perithecia spreads backwards from the line marking the start of the dark treatment.

In addition to perithecial zonation under single light-dark transfers, or under single light or dark exposures, these assumptions can also account for the zonation pattern found under $24 \mathrm{hr}$ light-dark alternating regime, as well as for zonation under regimes of shorter alternations (for a demonstration that these assumptions are sufficient see the Appendix below; it does not follow, however, that they are necessary). With respect to the findings in Fig. 2 we have already discussed the appearance of perithecial bands at the ends of light-grown sections with sharp cut-offs towards the boundaries with the dark-grown portions, obtained under $24 \mathrm{hr}$ alternating light and dark periods. Preliminary experiments were also done on $\mathrm{I} 2 \mathrm{hr}$ alternations, in which case a narrow perithecial band develops after each dark/light boundary, one every $24 \mathrm{hr}$. Similar experiments with $6 \mathrm{hr}$ alternating periods resulted in no bands of perithecia.

The assumptions listed above are reducible to two light-effects on perithecial initiation: the thioguanine-induced inhibitory effect of blue light, and the transient inhibition of perithecia following a light-to-dark transfer. The molecular mechanism of the first effect poses interesting questions. First of all, by analogy to the in vitro 
destruction of thioguanine in the presence of photosensitizing dyes (Friedman, 1968), a blue-absorbing compound must be present in the cells having a similar function. The two main classes of compounds with absorption in the blue region are the carotenoids and the flavins. The carotenoids seem to be ruled out, since perithecial inhibition can be observed when dark-grown cultures are exposed to I hr of light, and since the formation of carotenoids itself must be induced by light, therefore, there does not seem to be sufficient time for the newly produced pigments to act on thioguanine. This leaves the flavins and flavoproteins as the most likely photosensitizing pigments, and their role is planned to be further investigated. Flavoproteins are the most likely candidates for many of the blue-sensitive reactions in fungi, including the induction of carotenoid synthesis (Zalokar, I955; Rau, 1967).

The other question concerning the thioguanine effect is how the photo-destruction of thioguanine results in the inhibition of perithecial production but not of growth. Thioguanine may be decomposed either before it gets incorporated into nucleic acids, or after that, but in both cases the resulting changes should be equally deleterious to vegetative metabolic processes as to reproductive ones. A possible explanation may be that a certain kind of guanine-containing compound, which is essential for the initiation of perithecia, is more likely to come in contact with the photo-sensitizing pigment than the other nucleic acids. But it cannot be simply assumed that this compound essential for perithecia is a newly synthesized messenger RNA, since then all other newly synthesized messenger RNAs which are necessary for continued vegetative growth would also be inactivated. The compound involved in perithecial initiation must somehow be more exposed to photo-inactivation than the other guanine-containing cell components.

This investigation was supported by a grant to A.L. from the U.S. National Institutes of Health (GM I2547).

\section{APPENDIX}

\section{A. Lindenmayer}

The simple automata theoretical model which is presented here employs the principles for systems with state transitions and input-output relationships which are discussed in detail by Ashby (1956), and which have been applied to the development of filamentous organisms (Lindenmayer, 1968). The Sordaria colony growing in a growth tube is treated as a filament of discrete segments which undergo changes of state in discrete time units according to the light or dark conditions to which they are exposed. The filament is considered as growing only at its apex, $6 \mathrm{hr}$ of growth is arbitrarily chosen as the unit segment, and $6 \mathrm{hr}$ intervals as the time units. The state of a segment in a given interval is determined by its state and its input (light or darkness) in the previous interval. The following states and inputs are assumed:

States: $P$-segment determined to produce perithecia, $N$-segment determined not to produce any perithecia, I, 2, 3, 4-transitional dark-grown states, 5, 6, 7, 8transitional light-grown states.

Inputs: $L$-light treatment, $D$-dark treatment.

The apical segment is assumed to give rise to another segment of equal length every $6 \mathrm{hr}$. In other words, we are assuming a constant rate of growth, independent of the light or dark input. 
On the basis of the four assumptions listed in the Discussion for thioguaninecontaining cultures, we construct the following transition matrix:

$$
\begin{array}{lclllrlll} 
& \text { I } & 2 & 3 & 4 & 5 & 6 & 7 & 8 \\
L & 65 & N & 8 & 7 & 65 & 7 & 8 & N \\
D & 2 \mathrm{I} & 3 & 4 & P & N \mathrm{I} & 2 & 3 & 4
\end{array}
$$

This transition matrix specifies a next state or pair of states for each intermediate state and each input. $P$ and $N$ are terminal states; once a segment is in one of these, it does not change any further. Where the value of the matrix is a pair of states, we are dealing with the growth of an apical segment. New segments always arise in states I or 5 , depending on the input; these are the two initial states with which all computations must be started. We can perform sequential computations for any light-dark regime in which the alternating periods are in units of $6 \mathrm{hr}$.

Under continuous light or darkness the sequence of computed arrays will be very simple (the input shown next to the array is the one used to compute the next array):
$L \quad 5$
$D$ I
$L \quad 65$
D 2 I
$L \quad 765$
D $32 \mathrm{I}$
$L \quad 8765$
D 432 I
$L \quad N 8765$
D $P 4321$
L $\quad N N 8765$
D P P 432 I

The progressions of intermediate states through which each segment passes may be thought of as processes of differentiation which result in 18 to $24 \mathrm{hr}$ in the determination of a segment for bearing perithecia or staying vegetative.

The developmental sequence under alternating $24 \mathrm{hr}$ periods of light and darkness is the following one:

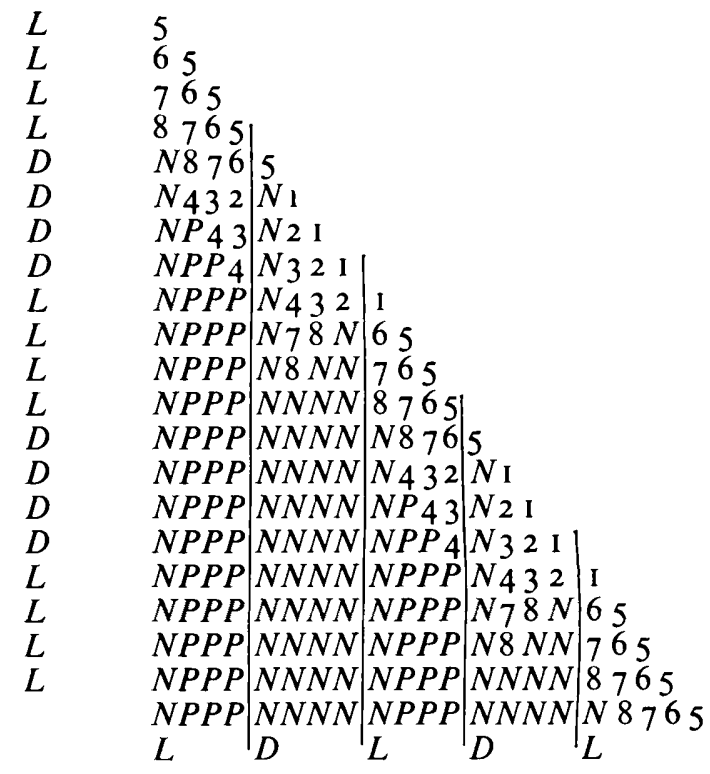


The vertical lines separate the sections which originally developed in light or darkness (marked $L$ or $D$ at the bottom). These lines correspond to the boundaries which were marked on the growth tubes as the daily increments of growth. In agreement with Fig. 2, the perithecial zones appear at the later portions of light-grown sections, and the last perithecial zone is in the last-but-one light-grown section.

Similar computations can be performed for each of the light-dark regimes used for the experiments shown in Fig. 3, producing the corresponding zonation patterns. Only the last line of the computation corresponding to tube $\mathrm{E}$ is shown here (cultures grown for several days in the dark, exposed to $24 \mathrm{hr}$ light, and returned to dark):

$$
\begin{array}{c|c|c}
\ldots P P P P P N N N & N P P P & N P P P P P 4321 \\
D & L & D
\end{array}
$$

It can also be demonstrated that light periods shorter than $24 \mathrm{hr}$ produce shorter zones of inhibition preceding the boundary of the light-grown section.

The zonation patterns discussed so far could be constructed on the basis of purely verbal assumptions and arguments. But it would be considerably harder to figure out intuitively what the pattern should be for alternating periods of less than $24 \mathrm{hr}$ duration, and for light and dark periods of unequal lengths. For example, what kind of zonation should we expect under an alternating $\mathrm{I} 2 \mathrm{hr}$ light- $\mathrm{I} 2 \mathrm{hr}$ dark regime? The answer worked out with our model turns out to be:

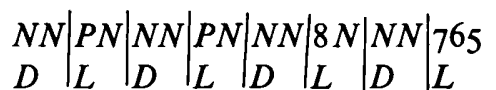

Narrow perithecial zones should develop following each dark-light boundary, and this is what was observed in a few experiments.

A similar question can be asked about alternating $6 \mathrm{hr}$ periods of light and dark. The result obtained from the model is that no perithecial zones should develop, and that some of the segments will actually cycle through transitional states indefinitely, The lack of perithecial zones was confirmed by experiment.

This model is, of course, only one of many such constructions which would account for these observations. The fact that it is a discrete model makes the computations much easier to perform than with a continuous model based on differential equations. One important characteristic of this model is that no interactions need to be assumed to take place among the segments, while other processes can only be simulated by assuming such interactions.

\section{REFERENCES}

Ashby, W. R. (1956). An Introduction to Cybernetics. London: Chapman and Hall. Bretzloff, C. W., JUN. (1954). The growth and fruiting of Sordaria fimicola. Am. J. Bot. 41, 58. Frifdman, P. A. (1968). Studies on the photodynamic reaction of purines and purine analogues with methylene blue. Biochim. biophys. Acta 166, I.

Goodwin, T. W. (1965). Chemistry and Biochemistry of Plant Pigments. New York: Academic Press. GreIS, H. (1936). Entwicklungsgeschichte von Sordaria fimicola. Bot. Arch. 38, I I 3.

HAWker, L. E. (1957). The Physiology of Reproduction in Fungi. Cambridge: University Press.

INGOld, C. T. \& Dring, V. J. (1957). An analysis of spore discharge in Sordaria. Ann. Bot. (N.S.) 21, 465.

Ingold, C. T. \& Hadland, S. A. (1959). Phototropism and pigment production in Sordaria in relation to quality of light. Ann. Bot. (N.S.) 23, 425 . 
Lindenmayer, A. (1968). Mathematical models for cellular interactions in development. I \& II. J. theor. Biol. 18, 280, 300.

LindenMayer, A. \& SCHOEN, H. F. (1967). Selective effects of purine and pyrimidine analogues and of respiratory inhibitors on perithecial development and branching in Sordaria. Pl. Physiol., Lancaster 42, 1059.

Nicolaus, R. A. (1968). Melanins. Paris: Hermann.

RAU, W. (1967). Light dependent carotenoid synthesis. I. Action spectrum of photoinduction in Fusarium aquaeductuum. Planta 72, I4.

ShapiRo, R. (1968). Chemistry of guanine and its biologically significant derivatives. In Progress in Nucleic Acid Research and Molecular Biology. Ed. by J. N. Davidson and W. E. Cohn. Vol. 8, p. 73. New York: Academic Press.

SURAPIPITH, V. (1969). Zonation in Sordaria fimicola and factors affecting its growth, perithecial initiation and pigment formation. M.A. thesis, Queens College, Flushing, N.Y.

WAlkeY, D. G. A. \& HARveY, R. (1967). Spore discharge rhythms in Pyrenomycetes. III. Ascospore production and the quantitative and qualitative influence of light on spore discharge in Sordaria macrospora. Trans. Br. mycol. Soc. 5o, $24 \mathrm{I}$.

ZaLOKAR, N. (1955). Biosynthesis of carotenoids in Neurospora. Action spectrum of photoactivation. Archs Biochem. Biophys. 56, 318. 